\title{
Estudio evaluativo del diseño e implementación curricular de la formación pedagógica en carreras de educación
}

An evaluative study of the design and curricular implementation in the pedagogical training in

Education Careers

\section{Volumen 17, Número 2 \\ Mayo-Agosto}

pp. 1-23

\author{
Fancy Castro Rubilar \\ Hugo Lira Ramos \\ María Teresa Castañeda
}

Revista indizada en REDALYC, SCIELO

Revista distribuida en las bases de datos:

LATINDEX, DOAJ, REDIB, IRESIE, CLASE, DIALNET, SHERPA/ROMEO, QUALIS-CAPES, MIAR

Revista registrada en los directorios:

ULRICH'S, REDIE, RINACE, OEI, MAESTROTECA, PREAL, $\underline{\text { CLACSO }}$ 


\title{
Estudio evaluativo del diseño e implementación curricular de la formación pedagógica en carreras de educación
}

An evaluative study of the design and curricular implementation in the pedagogical training in Education Careers

\author{
Fancy Castro Rubilar ${ }^{1}$ \\ Hugo Lira Ramos ${ }^{2}$ \\ María Teresa Castañeda ${ }^{3}$
}

\begin{abstract}
Resumen: El presente artículo describe los resultados del estudio que tuvo como objetivo evaluar el diseño, implementación y desarrollo de la estructura curricular de la formación pedagógica de las carreras de educación de una universidad pública, en el marco de la implementación de la formación por competencias en la educación superior en Chile. La metodología utilizada consideró un enfoque cualitativo y diseño de estudio de caso. La muestra estuvo conformada por 8 directores de carreras, 12 académicos y 64 estudiantes de las carreras señaladas. Para la recopilación de datos se empleó fuentes documentales, entrevistas semi-estructuradas y grupos de discusión. Como técnica de análisis se optó por Análisis de Contenido temático mediante software QDA. Los hallazgos obtenidos indican la falta de conexión entre los distintos componentes del diseño curricular investigado, evidenciando inconsistencias estructurales y operativas. Las principales conclusiones advierten que los componentes curriculares de la formación pedagógica se estructuran de manera aislada en los procesos de renovación del currículum, evidenciando, principalmente, disonancias. Este estudio demostró que la propuesta curricular vigente, objeto de esta investigación, se configura escindida de los cambios y propósitos declarados. Esto plantea la necesidad de que las renovaciones curriculares vayan aparejadas de un sistema de gestión y monitoreo para la concreción y verificación de los procesos formativos, con el fin de detectar oportunamente las posibles inconsistencias o nudos críticos.
\end{abstract}

Palabras clave: evaluación, formación de profesores, investigación, currículo.

\begin{abstract}
The present article describes the results of the study that aimed to evaluate the design, implementation and development of the curricular structure of the pedagogical training of the educational careers of a public university, within the framework of the implementation of training by competences in the Higher Education in Chile. The methodology used considered a qualitative approach and case study design. The sample consisted of eight race directors, 12 academics and 64 students of the indicated careers. Documentary sources, semi-structured interviews and discussion groups were used for data collection. As analytical technique, thematic Content Analysis was chosen using QDA software. The obtained results indicate the lack of connection between the different components of the curricular design investigated, evidencing structural and operational inconsistencies. The main conclusions indicate that the curricular components of the pedagogical training are structured in an isolated way in the processes of renewal of the curriculum, evidencing, mainly, dissonances. This study demonstrated that the current curricular proposal, object of this research, is configured split of the declared changes and purposes. This raises the need for curricular renewals to be coupled with a management and monitoring system for the concretion and verification of training processes, in order to detect in a timely manner possible inconsistencies or critical nodes.
\end{abstract}

Keywords: Evaluation, teacher training, investigation, curriculum.

\footnotetext{
${ }^{1}$ Académica Universidad del Bío-Bío, Chile. Profesora de Estado en Castellano. Doctora en Diseño Curricular y Evaluación Educacional, Universidad de Valladolid, España. Dirección electrónica: fcastro@ubiobio.cl
}

2 Académico Universidad del Bío-Bío, Chile. Psicopedagogo. Magíster en Orientación Educacional, Universidad de Playa Ancha de Ciencias de la Educación. Dirección electrónica: $\underline{\text { hlira@ubiobio.cl }}$

${ }^{3}$ Académica Universidad del Bío-Bío, Chile. Profesora de Historia, Geografía y Educación Cívica. Magíster en Curriculum, Evaluación e Innovación Educativa, Universidad Complutense, España. Dirección electrónica: mcastane@ubiobio.cl

Artículo recibido: 21 de junio, 2016

Enviado a corrección: 9 de diciembre, 2016

Aprobado: 13 de febrero, 2017 


\section{Introducción}

El estudio desarrollado tuvo como objetivo evaluar el diseño, implementación y desarrollo de la estructura curricular de la formación pedagógica de las carreras de educación, en el contexto de los procesos de renovación curricular.

La renovación del currículum de formación de las distintas carreras profesionales, particularmente, la formación de profesores, se ha constituido en una exigencia asociada a las demandas de un mundo cambiante y al desarrollo de la sociedad del conocimiento. Se requiere cada vez más de un recurso humano capaz de movilizar conocimientos, habilidades y destrezas para actuar de manera competente en la resolución de problemas y en los diversos desafíos que plantean los nuevos contextos laborales.

En este marco, las renovaciones de las carreras de pedagogía no siempre se han desarrollado con la sistematicidad que se requiere, puesto que han surgido desde la urgencia impuesta, más que de un proceso legitimado por la participación social de los actores involucrados, careciendo muchas veces de la suficiente reflexión académica. Uno de los aspectos relevantes sobre los cuales se requiere información empírica es acerca de la coherencia interna del currículum, así como de la pertinencia con los procesos de cambio curricular. Para ello ha sido necesario focalizarse en los componentes que conforman la estructura del currículo de la formación pedagógica, tanto en su diseño curricular, implementación y desarrollo.

En el afán por declarar los cambios y las renovaciones curriculares demandadas, las instituciones en general $y$, en particular, el caso estudiado evidencian un hiato entre lo declarado, lo realizado y el modo en que se implementa. En este sentido, se observa escaso monitoreo sobre la coherencia de los componentes del diseño curricular, específicamente, entre los principios pedagógicos, el perfil de egreso y los programas de asignatura. Existen varios nudos críticos en los procesos de configuración de un plan de estudio renovado, entre ellos está el que los profesores formadores en general no participan o no adscriben a los cambios establecidos institucionalmente, de modo que en el espacio particular decisional del currículo y en la puesta en práctica de este no siempre acogen o asumen la renovación diseñada.

La falta de conocimiento y manejo en diseños curriculares por competencias es otro gran nudo crítico, donde se antepone una resistencia colectiva a un esquema curricular que suele ser asociado con la perspectiva ideológica del modelo económico neoliberal, con una tendencia a superponer lo técnico por sobre la formación humana de los sujetos. 
A lo anterior se agrega, que los rediseños o renovaciones curriculares, generalmente, no son precedidas de una investigación evaluativa. Por el contrario, si se evaluaran, se tendría más claridad con respecto a qué es necesario cambiar y qué es conveniente mantener en el plan de estudio. Las modificaciones son orientadas externamente, es decir, en muchos casos estos procesos son direccionados por los requerimientos de las entidades que los financian, como lo son los proyectos de Mejoramiento de la Calidad de la Educación Superior (Mecesup) y los Convenios de Desempeño, ambos programas dependientes del Ministerio de Educación de Chile.

Los supuestos que han orientado la investigación evidencian que el marco del diseño y desarrollo curricular de la formación pedagógica carece de correlato con los contenidos y propósitos pedagógicos de los programas de estudio de las distintas asignaturas que la conforman.

A continuación, se proporcionan antecedentes acerca de la formación de profesores en Chile, la evaluación del currículum como una corriente de estudio propia, del diseño curricular y su implementación, el diseño metodológico empleado en el estudio, los resultados, análisis alcanzados y las conclusiones finales.

\section{Breve referente teórico}

\subsection{Formación de profesores en Chile}

La formación de profesores en el país está constituida por niveles y modalidades relacionadas con la estructura del sistema escolar. Toma en consideración las asignaturas del currículum base, dando origen a distintas carreras de pedagogía, para formar docentes de educación parvularia, básica, especial y media. No obstante, en virtud de la autonomía universitaria las instituciones de educación superior son libres de diseñar sus propias carreras, como lo señala el Informe Comisión sobre Formación de profesores (Chile, Ministerio de Educación, 2005, p. 22). Ello ha traído como consecuencia que no exista la necesaria coordinación entre el Ministerio de Educación de Chile (Mineduc) y las universidades para analizar el currículum escolar. La Organization for Economic Cooperation and Development (OECD, 2004), en el contexto de la revisión de las políticas educacionales de Chile, indica la poca relación entre los currículos de formación inicial y el currículum escolar, existiendo una carencia de estudios más acuciosos sobre la materia. El grado de éxito o fracaso de las políticas públicas, de la formación de profesores, debería evaluarse en función a su contribución al mejoramiento de los resultados educativos de los 
estudiantes en el medio escolar y particularmente, de los estudiantes más vulnerables (Ortúzar, Flores, Milesi y Cox, 2009, p. 24).

\subsection{Políticas educacionales}

Durante la última década, las políticas de la educación chilena han buscado atraer, educar y apoyar al grupo docente capaz de enseñar a todo el alumnado a un nivel alto. El principio declarado demuestra que las políticas educacionales (Chile, Ministerio de Educación 1997 y 2015) vinculadas con el profesorado han estado en función del fortalecimiento a la profesión. Una estrategia multidimensional para profesionalizar la enseñanza ha sido incorporar incentivos con el fin de atraer postulantes de calidad a la profesión docente. En este sentido el Panel de Expertos para una Educación de Calidad (2010), en el informe final, proporciona datos reveladores sobre el aumento significativo de estudiantes de Pedagogía en la última década, por lo que manifiesta una seria preocupación por la calidad de gran parte de los programas de formación docente. En este contexto de mejoramiento de la formación profesional, cobran importancia los niveles de calidad de las instituciones formadoras, puesto que repercuten de manera directa sobre los resultados en su formación (Pedraja, Araneda, Rodríguez y Rodríguez, 2012).

Estudios sobre habilidades básicas de los estudiantes de Pedagogía (Larrondo, Lara, Figueroa, Rojas y Caro, 2007) y resultados de la Prueba Inicia ${ }^{4}$, evidencian un bajo desempeño de los estudiantes egresados en conocimientos pedagógicos y disciplinarios. A este respecto, Del Rio, Concha, Salas, Treviño y Walker (2013, p. 215) señala: "como parte de la solución se considera como factores estratégicos relevantes en la formación, el reclutamiento de futuros profesores, el monitoreo del proceso de formación, los requisitos y certificación para el ejercicio profesional docente". En este listado, no cabe duda que la clave principal es la formación del profesorado para asegurar la calidad requerida para la docencia.

En relación con lo anterior, son relevantes las decisiones que se toman en torno al currículum de formación, puesto que se establece el diseño o arquitectura curricular, que implica, entre otros, la selección, planificación de contenidos y su trayectoria. El diseño curricular es una forma de plasmar una intención educativa y se concibe como el conjunto de componentes relacionados entre sí de manera secuencial y organizada que permiten identificar contenidos, metodologías, cronogramas y secuenciación de las acciones de

\footnotetext{
${ }^{4}$ Es una prueba que se aplica al término del proceso de formación en las carreras de Pedagogía, de manera voluntaria para los estudiantes.
} 
enseñanza y aprendizaje en una institución (Maldonado, 2002). La concepción de diseño curricular, denominado por competencias considera más componentes, como son el perfil de egreso, en algunos casos el perfil de ingreso, los resultados de aprendizaje, entre otros, y las fuentes curriculares, a su vez exceden el espacio académico y se extiende a empleadores, egresados, estudiantes, además, de velar por la articulación e integración de cada uno de los elementos. Esto conlleva que la actividad de selección de contenidos se realice en virtud de aquello que es esencial para vivir en la sociedad y desempeñarse en ella, lo cual implica elegir actitudes, habilidades y conocimientos básicos. El enfoque por competencias insertado en el diseño considera los requerimientos del mundo laboral profesional como también los grandes problemas de la sociedad y de la investigación (Tobón, Rial, Carretero y García, 2006, p. 120).

\subsection{Estructura curricular}

La estructura del currículum de las carreras asociadas a la formación de educadores en América Latina, según el Tuning América Latina (2008) ofrece una compleja diversidad en relación con los planes y programas en términos de carga horaria, énfasis, contenidos y nombres de las disciplinas. Asimismo, esta diversidad se replica en cada país e internamente en cada universidad, como también entre los diseños curriculares de las diversas carreras.

A nivel nacional, la organización del currículum que predomina es la que consigna: formación general, formación de especialidad, formación profesional y formación práctica (Ávalos, 2014). El informe de la Comisión sobre Formación de profesores (Chile, Ministerio de Educación, 2005, p. 33) recomendaba lograr un adecuado equilibrio y articulación entre contenidos de formación general, de formación pedagógica y de la especialidad o dominio disciplinario correspondiente. Para ello, es fundamental determinar qué y cuánto de la disciplina es necesario manejar para enseñar en los distintos niveles del sistema.

\subsection{Competencias y estándares de formación}

La implementación del curriculum por competencias ha traído consigo una nueva forma de diseñar los planes de estudio de las carreras en la educación superior. Lo usual era que se diseñara el currículum al interior del claustro académico, con prescindencia de otros actores del proceso, pero lo más relevante de este enfoque es el énfasis en el desarrollo de conocimientos teóricos movilizados en capacidades y habilidades concretas, evidenciadas en el desempeño profesional. 
La exigencia de imprimirle mayor calidad a la formación de docentes en el país ha significado la intervención del enfoque curricular por competencias y una serie de iniciativas relacionadas con su aseguramiento. La Comisión Nacional de Acreditación CNA-Chile (2007) establecía, entre el 2007 y el 2009, criterios de evaluación de las carreras de educación, donde se definía el perfil del profesional de la siguiente manera:

La calidad docente de los egresados se demuestra en primer lugar cuando los educadores y educadoras disponen de los conocimientos y de las capacidades que les permitan enfrentar las tareas y resolver los problemas propios de su esfera de trabajo. Pero, en forma más importante, la calidad docente se demuestra cuando los educadores ofrecen evidencia de uso en su desempeño docente de ese conocimiento y capacidades. Para juzgar la calidad de la evidencia ofrecida es necesario disponer de criterios sobre aquello, que en un contexto de significaciones y valores compartidos sobre la educación y la docencia, consideramos como una práctica docente ejemplar.

Estas aseveraciones tienen su correlato en los estándares orientadores para carreras de Pedagogía del Ministerio de Educación de Chile, los cuales tienen una doble función: señalan un "qué", referido a un conjunto de aspectos o dimensiones que se debieran observar en el desempeño del futuro profesor o profesora; también, establecen un "cuánto" o medida, el cual permite evaluar qué tan lejos o cerca se encuentra un nuevo profesor o profesora de alcanzar determinado desempeño (Chile, Ministerio de Educación, 2010, p. 7).

Asimismo, el país ha emprendido el proceso de definir un marco de cualificaciones para la educación superior, que es un instrumento para el desarrollo, la clasificación y el reconocimiento de destrezas, conocimientos y competencias a lo largo de un continuo de niveles acordados (Tuck, 2007). Es considerada una vía para estructurar cualificaciones existentes y nuevas que se definen a partir de resultados de aprendizaje, es decir, afirmaciones claras acerca de lo que el estudiante debe saber o ser capaz de hacer, ya sea que haya sido aprendido en una sala de clases o en el lugar de trabajo. Este esfuerzo va en la dirección de contar con un instrumento organizador del sistema de educación, pues permite: beneficiar la articulación de un sistema de educación, favorecer el desarrollo de estándares de calidad para los distintos niveles formativos, fortalecer la transparencia y legibilidad de las cualificaciones y certificaciones ofrecidas por las instituciones de educación 
superior e incrementar la coherencia del sistema, reconociendo las diferencias entre los niveles, según Kri et al. (2013).

\subsection{Evaluación curricular}

En este escenario, se agrega otro desafío como es la necesidad de evaluar el currículum de formación, con la finalidad de dar paso a la mejora de los procesos formativos, así como de ponerlos en sintonía con las necesidades y demandas del medio. La evaluación curricular considera en su ámbito dos campos de conocimiento: currículum y evaluación, que si bien son producto de la respuesta que los expertos dieron a los problemas de la educación en la era industrial (Díaz, 1994), su desarrollo es muy joven en el campo de las ciencias de la educación. La evaluación curricular tiene una notable vinculación con el avance en los diversos aspectos del currículum y es una pieza clave para su validación (Casarini, 2004). En este proceso se considera una dimensión interna y una externa. En la interna se analizan diversos aspectos de la estructura y organización del plan de estudios, así como los datos que se obtienen del rendimiento académico. De esta manera, aspectos como fundamentos del plan, su vigencia, congruencia, continuidad y viabilidad, entre otros permiten dar cuenta de la primera dimensión. Esta dimensión se complementa, además, con otros datos provenientes del perfil académico y desempeño docente, así como del rendimiento académico por líneas de conocimiento, de acuerdo con los procesos enseñanza y aprendizaje, vinculado con el perfil socioeconómico del estudiante.

La dimensión interna busca determinar la coherencia de sus procesos, en tanto, la dimensión externa busca una correspondencia con la primera, dado que no son procesos excluyentes, sino, por el contrario, implican una perspectiva integradora y multidimensional de la complejidad del proceso educativo. Lo externo, en particular, considera las visiones de los empleadores, egresados y otros actores del medio laboral, así como otros referentes del sistema social.

A la par de definir las dimensiones internas y externas del currículum, este se puede analizar desde tres perspectivas: el currículum formal, el currículum real, y el currículum oculto. En este sentido, Casarini (2004, p. 7) señala que "El currículum formal (o plan de estudios) es la planeación del proceso de enseñanza-aprendizaje con sus correspondientes finalidades y, condiciones académico- administrativas". El currículum real (o vivido), según este autor, es la puesta en práctica del currículum formal con las inevitables y necesarias modificaciones que requiere la contrastación y ajuste entre un plan curricular y la realidad del 
aula. No habrá que olvidar que el currículum real encuentra su razón de ser en la práctica educativa. En tanto, el currículum oculto plantea que: "es una categoría de análisis que nos permite interpretar con mayor precisión la tensión existente siempre entre intenciones y realidad, entre currículum formal y currículum real” (Casarini, 2004, p. 9).

\subsection{Desarrollo curricular}

El desarrollo del currículum hace referencia a la puesta en práctica del proyecto curricular: la implementación del currículum sirve para retroalimentar, rectificar y ratificar el diseño, y así ajustar progresivamente el currículum formal al currículum real pero tratando, al mismo tiempo, de tender al logro del currículum formal a medida que el diseño se ajusta y modifica (Casarini, 2004). La tendencia y los estudios desarrollados en relación con los procesos de innovación curricular dan cuenta de que muchos de estos han avanzado en la dirección del diseño, pero muy pocos en su implementación. Sin duda, falta desarrollar institucionalmente la cultura de la innovación, que permita poner en práctica el currículum diseñado. La cultura de la innovación debe ser el valor académico más importante porque define la pertinencia de la educación superior a partir del servicio que presta a la sociedad pero, al mismo tiempo, la innovación requiere modificar la organización administrativa de las instituciones de tal forma que los cambios propuestos sean factibles (Herrera y Didriksson, 1999).

\subsection{Enseñanza universitaria}

En este contexto, la formación de los docentes debe considerar, también, la revisión de la práctica pedagógica de la educación superior en relación con la didáctica y la evaluación de aprendizajes, como dos componentes claves. Enseñar se hace cada vez más desafiante para los educadores en cualquiera de los niveles, dado que los aprendizajes que desarrollen los estudiantes dependerán de la calidad y creatividad de las oportunidades y experiencias que procure el profesor o profesora para construir conocimiento efectivo y significativo. En el escenario de la educación superior, es aconsejable analizar lo que conlleva una didáctica para este nivel, ya que el objeto de la didáctica tiene una naturaleza distinta en relación con quiénes son los que aprenden, por ello algunos autores la han denominado didáctica universitaria, según Agustín de la Herrán (2001).

Cuando el proceso educativo se focaliza en el sujeto que aprende, como activo y consciente protagonista de su formación, se hace insuficiente la didáctica asimilada solo al 
enseñar. Cada vez más se reconoce la importancia que sea un proceso dialógico que permite el reconocimiento legítimo del estudiante con su universo propio, pleno de creencias y de condiciones singulares. Al respecto, Moreno (2011, p. 32) señala que "hoy se sabe que existen diferentes tipos de talentos (o inteligencias) distribuidos de forma más o menos similar entre la población y que los individuos, según las oportunidades que tengan, desplegarán más unos talentos que otros". De acuerdo con esta perspectiva, habrá que preguntarse cómo la universidad (en este caso) puede aprovechar las capacidades de los estudiantes para diseñar experiencias $u$ oportunidades de aprendizaje que incrementen mejor sus talentos. Asimismo, este autor describe la didáctica

como una ciencia teórica-práctica: trata el qué, cómo y cuándo enseñar, su argumentación refrenda lo que se ha sostenido ampliamente que es que la teoría necesita de la práctica, porque es en ella donde se revalida y la práctica, a su vez, se nutre de la teoría. (p. 35)

Resulta relevante coincidir con Contreras (1994), quien plantea que la didáctica se ocupa de la enseñanza, y que es, en última instancia, una práctica humana que compromete moralmente a quien la realiza y es una práctica social; es decir, responde a necesidades, funciones y delimitaciones que están más allá de las intenciones y previsiones individuales de sus actores directos, necesitando atender a las estructuras sociales y a su funcionamiento para poder comprender su sentido total.

En este contexto de la educación superior, se revelan considerables tensiones en el proceso pedagógico relativas a la evaluación de los aprendizajes. Los estudiantes siempre manifiestan incertidumbre al respecto, porque las prácticas evaluativas ocurren generalmente de manera poco clara. La realidad de muchos estudiantes indica que, al momento de enfrentar la evaluación, la ven como un hecho punitivo y misterioso, en parte, porque tradicionalmente ha prevalecido la idea de que la evaluación es solo calificación, un episodio, que ocurre en un momento determinado y distinto al proceso de enseñanza aprendizaje.

La evaluación constituye un constructo que apoya el desarrollo didáctico, puesto que aporta importante información al proceso y es también una oportunidad de mejora. En este sentido, Himmel (2011) refiere a la evaluación como: un proceso compuesto por acciones, que tienen como propósito contribuir, en forma permanente al aprendizaje estudiantil, con el fin de promoverlo, de incrementar la habilidad de autoaprendizaje, mejorar su calidad estimulando la meta-cognición y motivar a los futuros graduados a seguir aprendiendo a lo 
largo de toda la vida (Himmel, 2011, p. 202). La didáctica y la evaluación en la formación de profesores requieren no solo ser dispositivos pedagógicos claves en el proceso, sino también ser considerados como una instancia de modelamiento para la práctica de los futuros docentes.

Las fuentes teóricas analizadas coinciden en la importancia de que los diseños curriculares, particularmente, en la formación pedagógica deben articularse de forma sinérgica para lograr una coherencia interna que permita un desarrollo del currículum en sus diversos niveles de concreción, asegurando su calidad. Esto implica un proceso de evaluación permanente de su diseño e implementación, para generar sintonía con los requerimientos del contexto, de las políticas educacionales y el enfoque por competencias.

\section{Metodología}

El diseño investigativo de estudio de caso consideró el plan de formación de carreras de educación, a partir de las cuales se analizan y evalúan los componentes del currículum con diversas técnicas. El énfasis metodológico estuvo en la dimensión cualitativa, empleándose de manera complementaria las técnicas de corte cuantitativa, como lo indica la tabla 1. 
Tabla 1. Plan de análisis de información

\begin{tabular}{|c|c|c|c|}
\hline Categorías & Componentes & $\begin{array}{l}\text { Procedimientos } \\
\text { /Informantes }\end{array}$ & Subcategorías \\
\hline \multirow[t]{6}{*}{$\begin{array}{l}\text { - Coherencia interna } \\
\text { entre los diferentes } \\
\text { componentes de la } \\
\text { estructura curricular }\end{array}$} & \multirow[t]{3}{*}{$\begin{array}{l}\text { Currículum } \\
\text { formal }\end{array}$} & \multirow{3}{*}{$\begin{array}{l}\text { Análisis documental: } \\
\text { Documentos del } \\
\text { plan de formación } \\
\text { pedagógica }\end{array}$} & $\begin{array}{l}\text { Principios: son las grandes } \\
\text { orientaciones pedagógicas que } \\
\text { deben permear todo el proceso } \\
\text { de formación. }\end{array}$ \\
\hline & & & $\begin{array}{l}\text { Perfil de egreso: Describe el } \\
\text { conjunto de competencias que } \\
\text { el estudiante debe poseer al } \\
\text { finalizar su proceso de } \\
\text { formación }\end{array}$ \\
\hline & & & $\begin{array}{l}\text { Plan de estudio: Es la } \\
\text { organización de las asignaturas, } \\
\text { particularmente, de la línea de } \\
\text { pedagógica en que se } \\
\text { materializa el proceso de } \\
\text { formación. }\end{array}$ \\
\hline & \multirow[t]{9}{*}{$\begin{array}{ll}\text { Diseño } & \text { y } \\
\text { gestión } & \\
\text { curricular } & \end{array}$} & \multirow{9}{*}{$\begin{array}{l}\text { Entrevista semi } \\
\text { estructurada: } \\
\text { Directores de } \\
\text { carrera } \\
\text { Docentes de la } \\
\text { formación } \\
\text { pedagógica }\end{array}$} & $\begin{array}{l}\text { Verificación de principios: nivel } \\
\text { de conocimiento acerca de los } \\
\text { principios pedagógicos. }\end{array}$ \\
\hline & & & $\begin{array}{l}\text { Valoración de los principios: } \\
\text { vinculación con las acciones en } \\
\text { la gestión del currículum de la } \\
\text { carrera. }\end{array}$ \\
\hline & & & $\begin{array}{l}\text { Verificación de perfil: nivel de } \\
\text { conocimiento y desarrollo de las } \\
\text { competencias declaradas }\end{array}$ \\
\hline \multirow[t]{6}{*}{$\begin{array}{lr}\text {-Coherencia de la } \\
\text { implementación } & y \\
\text { desarrollo curricular de } \\
\text { la formación pedagógica }\end{array}$} & & & $\begin{array}{l}\text { Monitoreo del Perfil: revisión } \\
\text { sistemática del proceso de } \\
\text { aplicación del perfil en las } \\
\text { asignaturas. }\end{array}$ \\
\hline & & & $\begin{array}{l}\text { Concepciones didácticas: ideas } \\
\text { que posee el profesorado del } \\
\text { proceso de enseñanza y } \\
\text { aprendizaje. }\end{array}$ \\
\hline & & & $\begin{array}{l}\text { Práctica de la enseñanza y } \\
\text { aprendizaje: percepciones y } \\
\text { creencias que poseen el cuerpo } \\
\text { docente. }\end{array}$ \\
\hline & & & $\begin{array}{l}\text { Planificación de la enseñanza: } \\
\text { selección y organización de los } \\
\text { componentes curriculares. }\end{array}$ \\
\hline & & & $\begin{array}{l}\text { Aplicación de estrategias de } \\
\text { enseñanza y aprendizaje: uso } \\
\text { de procedimientos didácticos }\end{array}$ \\
\hline & & & $\begin{array}{l}\text { Aplicación de procedimientos e } \\
\text { instrumentos de evaluación: } \\
\text { utilización de criterios y } \\
\text { modalidades de evaluación }\end{array}$ \\
\hline
\end{tabular}

Fuente: Elaboración propia a partir de los objetivos que guiaron la investigación. 
Se seleccionó, intencionadamente, ocho carreras de educación ${ }^{5}$ : Pedagogía en Castellano y Comunicación, Historia y Geografía, Inglés, Educación Parvularia, Educación Física, Educación Matemática, Educación General Básica y Ciencias Naturales. Estas se constituyeron en fuente de información a partir del estudio a 8 directores, 12 académicos, que dictan las asignaturas de la formación pedagógica, y a la muestra azarosa de 64 de estudiantes de un total de 310, que cursaban el sexto semestre en las respectivas carreras.

Los procedimientos y técnicas empleados para la recopilación de información fueron: el análisis documental de los componentes de la propuesta curricular (principios pedagógicos, perfil de egreso y programas de asignatura), la entrevista semiestructurada para directores de carrera y para docentes de la formación pedagógica y, por último, grupos de discusión, con guía temática para generar el diálogo con los estudiantes seleccionados. La validación de los instrumentos empleados en este estudio se efectúo mediante el juicio de expertos, que consideró a académicos especialistas en evaluación y en currículum.

La confirmación metodológica de la evaluación curricular fue dada por la triangulación de los instrumentos empleados, cuya combinación estuvo dirigida a ofrecer una mirada cuantitativa y cualitativa, tanto de la recurrencia y grados de coherencia entre las categorías temáticas estudiadas, como de su naturaleza interpretativa.

Desde una perspectiva cuantitativa, se determinó la coherencia interna, entendida como el grado de relación existente entre los diferentes componentes de la estructura curricular estudiada, mediante análisis de frecuencias. Desde una perspectiva cualitativa, se analizó el corpus generado a partir de la desgravación y transcripción literal de las entrevistas y grupos de discusión aplicada, mediante el software de análisis cualitativo QDA, versión 1.01, a partir de las categorías de análisis señaladas en la Tabla del plan de análisis de información.

\section{Resultados y su análisis}

Los resultados y análisis de este estudio responden al objetivo principal, evaluar el diseño, implementación y desarrollo de la estructura curricular de la formación pedagógica de las carreras de educación. Este considera dos categorías centrales: coherencia interna entre los diferentes componentes de la estructura curricular y coherencia de la implementación y desarrollo curricular de la formación pedagógica.

\footnotetext{
${ }^{5}$ Este artículo está basado en los resultados de una investigación financiada por la Dirección de Investigación de la Universidad del Bío Bío, Chile, bajo el código N 063622 3/R.
} 
El análisis documental consideró "Perfil académico y profesional de los egresados, 2005" y el documento "Diseño de las mallas curriculares de las carreras de pedagogía, 2005", ambos textos vigentes en la formación pedagógica del caso estudiado. El plan de análisis documental consideró dos categorías específicas: la coherencia entre principios orientadores de la formación inicial y el perfil de egreso de las carreras de Pedagogía y la coherencia entre el perfil de egreso y los programas de asignaturas del área de formación pedagógica. En este marco se estableció la noción de coherencia como la categoría sustancial del estudio, que se refiere a la articulación existente entre los diferentes componentes curriculares, tanto a nivel conceptual, procedimental y actitudinal.

La primera categoría, coherencia interna entre los diferentes componentes de la estructura curricular, en ese marco el concepto de principio es entendido, en este estudio, como eje orientador de la readecuación de las mallas curriculares a las nuevas demandas del contexto y considera los principios de: inclusión, vinculación de la teoría y práctica, saber pedagógico, cultura indagativa, desarrollo personal, vinculación escuela y comunicad e incorporación a las tecnologías de la información y comunicación. En tanto, el perfil de egreso es un referente que orienta la formación de un profesional de la educación autónomo, con capacidad de reflexionar críticamente acerca de su práctica, fuertemente vinculado al contexto y aportando a la formación de equipos y al desarrollo de las instituciones, las competencias que lo forman son: actúa comprometido con las personas y su contexto, comunica en forma clara y precisa los contenidos de su campo disciplinario, contextualiza el proceso de enseñanza y aprendizaje, evalúa críticamente el proceso y el producto de la enseñanza, asume una actitud indagativa y problematizadora en la práctica pedagógica, asume sus responsabilidades profesionales, trabaja en equipos interdisciplinarios, adopta una actitud abierta y crítica frente a los cambios, crea un ambiente propicio para el aprendizaje y actúa con principios éticos.

El análisis arrojó que el principio de inclusión, que implica la inclusión social y educativa de los sujetos, logra una coherencia de un $40 \%$ de las competencias señaladas en el perfil, siendo una de las más altas encontradas en el análisis documental. El principio de vinculación teoría y práctica, que define esta vinculación como un proceso dialéctico en que la teoría ilumina a la práctica como a su vez la práctica ilumina a la teoría, presenta una baja coherencia, la que alcanza un $10 \%$ con las competencias señaladas en el perfil de egreso. El principio del saber pedagógico, que se considera como el saber que el docente genera en la práctica pedagógica y que se constituye en un constructor determinado por la 
interacción entre el conocimiento teórico y la realidad histórica, social y cultural, en la que desarrolla su acción educativa, alcanza una coherencia de $40 \%$ con las competencias del perfil. El principio de la cultura indagativa, que implica desarrollar una actitud de búsqueda de nuevas y creativas respuestas a las problemáticas que emergen de la práctica pedagógica cotidiana, alcanza solo un $20 \%$ de coherencia con el perfil de egreso. El principio del desarrollo personal, implica formar un pedagogo con una alta valoración de sí mismo, de su profesión y de su función social y alcanza una coherencia de un $30 \%$, cuestión que se puede entender como un desafío emergente del pedagogo ligado a su responsabilidad como modelo de conducta. El principio de vinculación escuela y comunidad, hace referencia a la necesidad de que el pedagogo posea competencias para descubrir y comprender las necesidades e intereses reales de aquellos contextos sociales y culturales en los cuales le corresponda, presenta una coherencia del 30 \%. El principio de incorporación a las TIC, que sitúa al pedagogo en un nuevo escenario determinado por el desarrollo y la complejidad de las nuevas tecnologías, presenta una baja coherencia de este principio con el perfil de egreso, logrando solo un $10 \%$.

En síntesis, el promedio la coherencia entre los principios y las competencias del perfil de egreso alcanza aproximadamente un $25 \%$. Esta coherencia se estima baja para las expectativas de congruencia interna esperables para la formación pedagógica. Luego del análisis documental, las principales debilidades detectadas están relacionadas con el grado de materialización de los principios en las competencias del perfil; asimismo, se puede inferir que la construcción de los principios y de las competencias del perfil fueron procesos discontinuos, que operaron desarticuladamente, lo que condujo a una distancia evidente entre ambos constructos.

Se estableció que la representación de las competencias del perfil de egreso de la formación pedagógica en los programas de asignatura era predominantemente básica e insatisfactoria; por tanto, la coherencia entre estos dos componentes del currículo formal es muy baja. Se pudo establecer que de las diez competencias, solo las siguientes están relacionadas con temas de carácter transversal o genérico y, además, presentan un nivel de coherencia insatisfactorio: trabaja en equipos interdisciplinarios, asume responsabilidades profesionales, adopta una actitud abierta y crítica frente a los cambios, crea un ambiente propicio para el aprendizaje y actúa con principios éticos. Esto podría significar un escaso desarrollo de competencias genéricas y profesionales en la formación pedagógica, en contraste con lo declarado en el curriculum formal. En tanto, cinco competencias vinculadas 
a la dimensión pedagógica presentan un nivel de coherencia básico a nivel de currículum formal: la actuación comprometida con las personas y su contexto, comunica en forma clara y precisa los contenidos de su campo disciplinario, evalúa críticamente el proceso y el producto de la enseñanza, asume una actitud indagativa y problematizadora. Esta constatación es preocupante, si se considera que los profesores en ejercicio, que han sido evaluados en el marco de la evaluación docente en Chile desde el 2003 a la fecha, presentan serias deficiencias en la puesta en práctica en estas competencias.

La segunda categoría, coherencia de la implementación y desarrollo curricular de la formación pedagógica, permitió contrastar el currículum declarado y el curriculum desarrollado, por medio de entrevistas, estas posibilitaron la identificación del tipo de conocimiento que poseen los directores de carrera y docentes, en relación con los principios y perfil establecido en formación pedagógica. La información recopilada fue en relación con los principios, el perfil de egreso, las concepciones didácticas, la planificación de la enseñanza. Se pudo evidenciar que tanto los directores de carrera como los docentes de formación pedagógica, en una primera aproximación, dicen desconocer por completo dichos principios. A medida que se avanza en la entrevista, solo en el discurso de los docentes, se pudo inferir una valorización por algunos principios como: la inclusión, la vinculación de la teoría y la práctica, el saber pedagógico, la cultura indagativa y el desarrollo personal. Al presentarles a los docentes los principios por escrito, estos los jerarquizaron estableciendo principios mejor y menor desarrollados en el currículum.

Por una parte, los jefes de carreras entrevistados consideran relevantes estos principios para la formación profesional del docente, además, observan coincidencia con los principios propios de las especialidades de sus respectivas carreras. Por otra parte, los docentes valoraron, especialmente, los principios vinculados a lo cultural y el desarrollo personal. En este aspecto se visualiza una disonancia entre la valorización de los principios y su concreción en el currículum formal y real, dado que tienen una débil presencia y escasa intención de incorporarlos. En tanto, en el perfil de egreso se encontró que poseen un conocimiento completo de este, una minoría de directores de carrera y otros pocos dicen tener un conocimiento parcial de las competencias de este perfil. En el caso de los docentes, afirman no conocer las competencias y, por cuanto, difícilmente podría desarrollarlas en el contexto de su asignatura.

De las competencias trabajadas solo fue posible identificar las que hablan de una percepción de mejor desarrollo de la actitud indagativa, seguidamente las referidas al 
compromiso con la persona y el entorno, la actitud abierta y crítica, y en los últimos lugares, ambiente propicio para el aprendizaje, principios éticos y evaluación de la enseñanza. Dada la inconsistencia del conocimiento y el desarrollo de las competencias, se puede colegir que no hay competencias mejor desarrolladas, sino que están al nivel de declaraciones formales. Esta percepción se basa en que los únicos que dan cuenta de esta información son los jefes de carrera y no los docentes, que también deberían tener conocimiento de acuerdo con su función.

En cuanto a la verificación de la aplicación del perfil, los directores de carrera señalan que realizan una verificación parcial del perfil, obligados por los procesos de acreditación de las carreras. No se observa, de parte de los directores, un compromiso por monitorear el cumplimiento del perfil, la mayoría dice no realizar evaluación sistemática de este, solo algunas excepciones mínimas consignan realizar evaluación semestral a través de distintos procedimientos. En algunas carreras se ha ido instalando paulatinamente la práctica de establecer un portafolio semestral por asignatura, con el fin de sistematizar la información requerida para los procesos de acreditación, principalmente. Los docentes, por su parte, a partir del listado que se les presentó, distinguen entre las competencias aquellas consideradas como las más relevantes, destacando seis de ellas. Estas hacen referencia al compromiso local, la contextualización de la enseñanza y evaluación, el mejoramiento del currículum, la investigación pedagógica y, sobre todo, valoran el trabajo interdisciplinario. Los docentes proponen nuevas competencias al perfil, pero curiosamente aquellas que ya están establecidas, confirmando con ello el desconocimiento de las competencias vigentes.

Los directores dicen propiciar integración curricular al vincular los principios declarados y las competencias del perfil. La integración es apreciable, también, en la matriz curricular de cada carrera de Pedagogía, que incorpora la línea pedagógica y la línea disciplinaria, en la elaboración de los respectivos perfiles. Como ya se ha señalado, el análisis refleja que existe un deficiente dominio de las competencias de parte de los directores y de los docentes en relación con el conocimiento y con la incorporación de estas al desarrollo del currículum, tanto a nivel de gestión como a nivel asignatura.

En la subcategoría planificación de la enseñanza, los docentes señalan que la realizan semestralmente, pero asumiendo la posibilidad de realizar ajustes. Esta se centra en los contenidos, principalmente, sin considerar aspectos como las habilidades, destrezas, valores y las características de los estudiantes. En relación con los objetivos, afirman seleccionarlos a partir de los programas de asignatura dados, introduciendo algunas adecuaciones 
necesarias. Otros dicen considerar dimensiones conceptuales, procedimentales y actitudinales y que sí modifican los objetivos según las características del curso.

Con respecto a la subcategoría sobre concepciones didácticas que manejan los docentes, estos declararon asumir una perspectiva constructivista de la didáctica destacando su rol de mediador y la interpelación al estudiante por medio del diálogo. Señalaron que el proceso de aprendizaje que propician es activo, continuo y permanente, destacando en ello el aprendizaje significativo y el desarrollo de competencias, como perspectivas teóricos prácticas del citado proceso. En este mismo aspecto, dicen emplear estrategias de enseñanza, tales como: el trabajo en terreno, la motivación, la discusión, la exposición socializada y una práctica dialógica. En torno a la subcategoría recursos didácticos, se puede mencionar el uso de recursos tradicionales en coexistencia con los medios innovadores como son los tecnológicos.

La subcategoría práctica de enseñanza y aprendizaje, es entendida en este estudio, como las percepciones y creencias que poseen los docentes acerca de su propia práctica pedagógica. Estas están vinculadas con las dimensiones del aprendizaje, la valorización de la práctica, los criterios de evaluación y el ambiente social del aula. Al respecto, los docentes expresan que los estudiantes evalúan positivamente sus prácticas pedagógicas, principalmente, por su cercanía, disposición, metodología participativa, la experiencia y el conocimiento en su línea.

Los docentes dicen emplear diversidad de procedimientos evaluativos relacionados principalmente con actividades de aprendizaje, no obstante, la mayoría utiliza de manera recurrente instrumentos tradicionales (de tipo sumativo y final) y solo un grupo menor emplea procedimientos innovadores. Estos dicen utilizar criterios diversos para evaluar y señalan establecer preguntas vinculadas al conocimiento, comprensión, aplicación y análisis. El nivel taxonómico de análisis se considera como el criterio más empleado en la evaluación. En situaciones específicas, el grupo docente valora la participación, la asistencia, el compromiso y la responsabilidad, de manera transversal sin incidencia, necesariamente, en la calificación.

Producto de la triangulación de los grupos de discusión realizados con estudiantes surgieron categorías focalizadas en la aplicación de estrategias de enseñanza y aprendizaje. En ellas se han distinguido las dimensiones centradas en el recurso, en la interacción, en estudiante y docente. A este respecto, expresan que los docentes priorizan el uso del Power Point como uno de los medios más recurrentes, sin embargo, se critica su pasividad y el desaprovechamiento de este recurso. Asimismo, se valora el uso de tecnología como 
plataformas virtuales, el empleo de guías de aprendizaje, solo que en este último caso se dice que no aportan a la reflexión, debido a la falta de análisis; no obstante, dejan constancia de algunas excepciones.

Estudiantes coinciden con docentes cuando se valora la interacción como un aspecto clave para el aprendizaje, destacándose el debate como una estrategia que favorece la discusión y clarifica conceptos, como, también, el uso de trabajos grupales y talleres que propician el trabajo cooperativo; en este contexto, se aprecia la plataforma como un medio que posibilita las condiciones de interactividad.

En cuanto a las estrategias centradas en el estudiante, destacan los trabajos de investigación como una oportunidad de aprender de una manera más profunda, así como la lectura personal de textos dados. No obstante, critican los talleres que no se focalizan en el aprendizaje e identifican estrategias centradas en el docente, como las exposiciones de docentes donde no establecen interacciones y enfatizan excesivamente lo teórico, con algunas excepciones cuando presentan ejemplos que se vinculan con la vida diaria.

Los procedimientos e instrumentos de evaluación, según los estudiantes, son incoherentes con el proceso de enseñanza y aprendizaje en el aula, junto a ello plantean que no se consideran los distintos modos de aprender ni las opiniones personales. En general, se centran en la "lógica de la sorpresa" y se desarrollan en un tiempo insuficiente para generar una respuesta zanjada. Consideran, además, incongruente la alta ponderación dada a las pruebas escritas en relación con otros procedimientos evaluativos como el portafolio que, según estudiantes, contribuye más al aprendizaje profundo y exige un mayor esfuerzo en su construcción. No obstante, se observa cierta coincidencia con lo declarado por docentes cuando dicen que se utilizan preguntas de desarrollo abiertas, trabajos de investigación, uso de auto y coevaluación con rúbricas y la retroalimentación de resultados. No obstante, falta certeza que exista coherencia curricular con las competencias y habilidades declaradas en los perfiles de egreso, es decir, los escasos niveles de correspondencia logrados no son el fruto de un ejercicio sistemático o consciente de coherencia curricular y evaluativa (Möller y Gómez, 2014).

Otro aspecto destacable, según estudiantes, es que en algunas asignaturas se utilizan de manera alternada y variada los procedimientos e instrumentos de evaluación tanto de carácter tradicional como innovadores. En cuanto a los tradicionales, se continúa con la aplicación de pruebas escritas, disertaciones e informes grupales, en tanto, en los procedimientos innovadores se enfatiza en la necesidad del análisis de videos, aplicación de 
contenidos en la práctica, empleo de portafolios, la introducción de edublog, simulación de clases y debates.

Finalmente, el análisis documental como las respuestas dadas por los diversos informantes coincide en identificar inconsistencia tanto en lo declarado como en la práctica del currículum de formación pedagógica vigente en el caso estudiado.

\section{Conclusiones}

Este estudio permitió evidenciar que el currículum formal presenta disonancias internas entre sus componentes como son los principios pedagógicos, el perfil de egreso y los programas de asignatura, lo que desde la perspectiva de Casarini (2004) es un problema que desarticula el proceso formador. Sus consecuencias más directas se pueden observar en la planificación curricular, expresada en los programas de asignatura de la formación pedagógica, que no recogen las necesidades formativas del estudiante. Asimismo, en la implementación o desarrollo del currículum se demuestran disonancias cuando docentes caracterizan positivamente su docencia, a pesar que evidenciaron falta de conocimiento acerca de las competencias del perfil, lo que indica escaso análisis crítico acerca de su quehacer. Esto es refrendado por estudiantes, quienes identifican puntos conflictivos en la práctica docente, como lo es la poca eficiencia en el uso de los recursos didácticos, tanto físicos como virtuales, y recurrencia de estrategias didácticas y evaluativas de tipo tradicional. Estas alusiones a la práctica de los formadores del profesorado revelan varias debilidades, lo que confirma la necesidad de seguir profundizando la investigación acerca de la actuación pedagógica de docentes, porque ningún cambio es posible sin contar con el compromiso y mediación docente, "si bien este actor es el segundo en presencia dentro de todos los que atienden las investigaciones, observamos que se conceptualiza a un formador que hemos denominado 'genérico', y por lo mismo, sostenemos que es un actor estudiado desde aspectos muy generales" (Cisternas, 2011, p. 29).

Lo evidenciado, en esta investigación, por directivos y docentes de muestra el bajo compromiso por el diseño, implementación y desarrollo de la nueva estructura curricular de la formación pedagógica de las carreras de educación y su alineación con los procesos de renovación curricular realizados. A la ausencia de mecanismos de monitoreo sistemático del currículum se suma la falta de convicción de que las reformas son indispensables para lograr una educación más equitativa y de calidad (Panel de Expertos para una Educación de Calidad, 2010). Ello, también, indica que las instituciones no siempre proveen las 
condiciones necesarias para llevar a cabo la innovación curricular de las carreras, que el proceso no está concebido de manera sistémica. No se relacionan los componentes del currículum entre sí, además, falta coordinación de los actores del proceso, pues dejan en terreno incierto la puesta en acción del nuevo currículum diseñado.

La falta de consistencia encontrada no solo revela el fallo de conexión entre los distintos componentes curriculares del diseño investigado, sino que además este es inconsistente con su propia formalidad, dado que no se consideró en el diseño general las operaciones que lo ponen en práctica y que son necesarias para establecer su coherencia interna. Es por ello que la evaluación sobre el currículum prescrito y el currículum real dejó sentado la necesidad de que las renovaciones o rediseños curriculares vayan aparejados de un sistema de gestión del currículum, que permita entre otras estrategias, monitorear la concreción de los procesos formativos con el fin de detectar a tiempo las inconsistencias y los distintos nudos críticos. Los rediseños curriculares no siempre surgen de la necesidad detectada por medio de la evaluación; por el contrario, lo que ha predominado en estos procesos es la construcción curricular prescrita. Es por ello que se crea la incertidumbre acerca de que si lo que se cambia será para mejorar el plan de estudio o, por el contrario, la calidad se verá afectada.

Los resultados de esta investigación advierten y ponen en alerta el hecho de que los procesos de la renovación del plan de formación en sus diferentes aspectos no presentan coherencia curricular, porque no se generan de manera articulada. Se hace necesario, en consecuencia, configurar propuestas de renovación del currículum de manera estructurada, que permitan la fluidez y la evaluación permanente del proceso. Por lo tanto, debe ocuparse de desarrollar una cultura de la innovación, sostenida por la capacidad de convencer a todos los actores acerca de las virtudes y de la necesidad de instalar la práctica de que el diseño curricular de una carrera es una responsabilidad compartida, que no puede ser adjudicada a un equipo sino al conjunto de la comunidad relacionada con la formación de los futuros profesionales.

Luego del estudio realizado, se constata la existencia de variados aspectos que son necesarios de dilucidar en los procesos de renovación curricular, por tanto, esta se vuelve un espacio fértil para investigar y generar conocimiento actualizado, que aporte al mejoramiento cualitativo y cuantitativo para la formación de profesionales, particularmente, lo que dice relación con la formación de profesores, pues, la dinámica de los cambios sociales, políticos y pedagógicos, los ajustes o renovación del currículum de formación deben ser permanentes. 
Cuando se realizan ajustes curriculares se debe cautelar que lo definido en el diseño se lleve a cabo en la ejecución de este y su evaluación debe asumirse como un proceso con adecuadas estrategias e instrumentos para recoger observaciones e informaciones y analizarlas con la participación de los diversos actores implicados en él (Tovar y Sarmiento, 2011). No obstante, se hace necesario dejar sentado que la implementación de un proceso de renovación curricular no se agota solo en la evaluación, pues las acciones de la evaluación constituyen un factor para el mejoramiento de la educación. En la mayoría de los casos se atribuye a un resultado mecánico del ejercicio de la evaluación, sin estudiar las condiciones que se requieren para que la evaluación contribuya al mejoramiento del desempeño educativo. No es tan cierto que a mejores indicadores o mejores índices de evaluación se corresponda un mejor trabajo en el campo de la educación (Díaz, 2005). El estudio realizado deja planteada la necesidad de procurar la concurrencia articulada de los diferentes aspectos que intervienen en estos procesos, debido a la complejidad que involucra la renovación del currículum.

\section{Referencias}

Ávalos, Beatrice. (2014). La formación de profesores en Chile: Tensiones entre políticas de apoyo y control. Estudios pedagógicos, 40(Especial), 11-28. Recuperado de http://www.scielo.cl/scielo.php?script=sci arttext\&pid=S0718$07052014000200002 \& \operatorname{lng}=$ es\&tlng $=$ es

Casarini, Martha. (2004). Teoría y diseño Curricular. México: Trillas.

Cisternas, Tatiana. (2011). La investigación sobre formación docente en Chile. Territorios explorados e inexplorados. Revista Calidad en la Educación, (35), 131-164.

Comisión Nacional de Acreditación CNA-Chile. (2007). Criterios de Evaluación de Carreras de Educación (2007-2009). Recuperado de https://www.cnachile.cl/Criterios\%20de\%20carreras/CriteriosEducacionFINAL1.pdf

Contreras, José. (1994). Enseñanza, currículum y profesorado: introducción crítica a la didáctica. Madrid: Akal.

Chile, Ministerio de Educación. (1997). Estatuto de los Profesionales de la Educación. Santiago de Chile: Mineduc.

Chile, Ministerio de Educación. (2005). Informe Comisión Formación de profesores. Santiago de Chile, Serie Bicentenario: Mineduc. 
Chile, Ministerio de Educación. (2010). Estándares orientadores para las carreras de pedagogía en educación media. Santiago de Chile: LOM Ediciones Ltda. Recuperado de

http://portales.mineduc.cl/usuarios/cpeip/File/librosestandaresvale/libromediafinal.pdf

Chile, Ministerio de Educación. (2015). Sistema de Desarrollo Profesional Docente, Ley 20.903. Santiago de Chile: Mineduc.

Del Río, Francisca, Concha, Soledad, Salas, Natalia, Treviño, Ernesto y Walker, Horacio. (2013). Un modelo de monitoreo de los aprendizajes durante la formación de profesores: una oportunidad de contar con evidencia concreta de la calidad de los programas. Calidad en la educación, (39), 213-233. Recuperado de http://www.scielo.cl/scielo.php?script=sci arttext\&pid=S0718$45652013000200008 \& \operatorname{lng}=\mathrm{es} \& \operatorname{lng}=\mathrm{pt}$

Díaz, Ángel. (1994). El currículum escolar. Surgimiento y perspectivas. Buenos Aires: REIArgentina, IDEAS, Aique.

Díaz, Ángel. (2005). Evaluación curricular y evaluación de programas con fines de acreditación. Cercanías y desencuentros. Conferencia presentada en el Congreso Nacional de Investigación Educativa, Sonora, México. Recuperado de http://www.angeldiazbarriga.com/ponencias/conferencia cnie2005.pdf

Herrán, Agustín de la. (2001). Didáctica Universitaria: La Cara Dura de la Universidad. Tendencias Pedagógicas, (6), 11-38.

Herrera, Alma y Didriksson, Axel. (1999). La construcción curricular: innovación, flexibilidad y competencias. Educación Superior y Sociedad, 10(2), 29-52.

Himmel, Erika. (2011). Evaluación de aprendizajes en la educación superior: una reflexión necesaria. Pensamiento Educativo. Revista de Investigación Educacional Latinoamericana, 33(2), 199-211.

Kri, Fernanda, Marchant, Elisa, del Valle, Rodrigo, Sánchez, Tatiana, Altieri, Elena, Ibarra, Pamela, ... y Segovia, Nidia. (2013). Manual para la implementación del Sistema de Créditos Académicos Transferibles SCT-Chile. Santiago de Chile: Consejo de Rectores de las Universidades Chilenas, $\mathrm{CRUCH}$.

Larrondo, Tito, Lara, Marcela, Figueroa, Claudio, Rojas, María y Caro, Alberto. (2007). Desarrollo de habilidades básicas en lenguaje y matemáticas en egresados de pedagogía. Un estudio comparativo. Revista Calidad, (27), 150-176.

Maldonado, Miguel. (2002). Las competencias, una opción de vida: Metodología para el diseño curricular. Bogotá: Editorial Magisterio.

Möller, Isabel y Gómez, Héctor. (2014). Coherencia entre perfiles de egreso e instrumentos de evaluación en carreras de educación básica en Chile. Calidad en la educación, (41), 17-49. Recuperado de http://www.scielo.cl/scielo.php?script=sci arttext\&pid=S0718$45652014000200002 \& \operatorname{lng}=\mathrm{es} \& \ln \mathrm{n}=\mathrm{es}$ 
Moreno, Tiburcio (2011). Didáctica de la Educación Superior: nuevos desafíos en el siglo XXI. Revista Perspectiva Educacional. Formación de Profesores, 50(2), 26-54.

Organization for Economic Co-operation and Development (OECD). (2004). Reviews of National Policies for Education: Chile. Paris: OECD Publishing.

Ortúzar, Soledad, Flores, Carolina, Milesi, Carolina y Cox, Cristian. (2009). Aspectos de la formación de profesores y su influencia en el rendimiento académico de los alumnos. En Ignacio Irarrázaval, Elena Puga y Magdalena Letelier (Eds.) Camino al Bicentenario, propuestas para Chile, (pp.155-186). Santiago de Chile: Ediciones Universidad Católica.

Panel de Expertos para una Educación de Calidad. (2010). Propuestas para fortalecer la profesión docente en el sistema escolar chileno. Informe final: Primera Etapa. Santiago de Chile: Gobierno de Chile.

Pedraja, Liliana, Araneda, Carmen, Rodríguez, Emilio y Rodríguez, Juan. (2012). Calidad en la Formación de profesores: Evidencia Empírica en las Universidades Chilenas. La Serena, Chile. Revista Formación Universitaria, 5(4) ,15-26.

Tobón, Sergio, Rial, Antonio, Carretero, Miguel y García, Juan. (2006). Competencias, calidad y educación superior. Colombia: Alma Mater, Magisterio.

Tovar, María Clara y Sarmiento, Pedro. (2011). El diseño curricular, una responsabilidad compartida. Revista Colombia Médica. 42(4), 508-517, Recuperado de http://www.redalyc.org/articulo.oa?id=28321543012

Tuck, Ron. (2007). An Introductory Guide to National Qualifications Frameworks: Conceptual and Practical Issues for Policy Makers. Switzerland: International Labour Organization.

Tuning América Latina. (2008). Reflexiones y perspectivas de la Educación Superior en América Latina. España: Publicaciones de la Universidad de Deusto. 